\title{
Consistency of shared reference frames should be reexamined
}

\author{
Fei Gao ${ }^{1,2 *}, \quad$ Fen-Zhuo Guo ${ }^{1}, \quad$ Qiao-Yan $\mathrm{Wen}^{1}$, and Fu-Chen $\mathrm{Zhu}^{3}$ \\ ${ }^{1}$ State key Laboratory of Networking and Switching Technology, Beijing University of \\ Posts and Telecommunications, Beijing 100876, China \\ ${ }^{2}$ School of Science, Beijing University of Posts and Telecommunications, Beijing 100876, China \\ ${ }^{3}$ National Laboratory for Modern Communications, P.O.Box 810, Chengdu 610041, China
}

\begin{abstract}
In a recent Letter [G. Chiribella et al., Phys. Rev. Lett. 98, 120501 (2007)], four protocols were proposed to secretly transmit a reference frame. Here We point out that in these protocols an eavesdropper can change the transmitted reference frame without being detected, which means the consistency of the shared reference frames should be reexamined. The way to check the above consistency is discussed. It is shown that this problem is quite different from that in previous protocols of quantum cryptography.
\end{abstract}

PACS numbers: 03.67.Dd, 03.67.Hk, 03.65.Ud

As we know, reference frame (RF) [1] is a kind of unspeakable information and consequently sharing of an RF is generally more difficult than that of a string of key bits as performed in a quantum key distribution (QKD) protocol [2]. In a recent Letter, Chiribella et al. proposed four quantum-cryptographic protocols to secretly communicate an RF [3]. These protocols are subtly designed so that the eavesdropper (say Eve) cannot obtain any information about the RF when it is transmitted between the users (say Alice and Bob). Here, from a different perspective of security, we consider a special threat which was not concerned in Ref. 3]. That is, after the communication, the consistency of the RF Alice sent with that Bob received is still not assured. We will show that by a special attack Eve can destroy this consistency without introducing any detectable disturbance.

Let us take the first protocol in Ref. [3] as our example, where Alice can transmit a secret direction ( $z$ axis) to Bob with the help of a string of secret key bits shared between them. Alice sends a sequence of spin- $\frac{1}{2}$ particles to Bob, whose particular states, i.e. spin up or down, are determined by the shared key bits 0 or 1 , respectively. After receiving this sequence, Bob measures the particles alternately along his own $x, y$, and $z$ axes and compares the measurement results with the shared key bits. By calculating the error rates associate to the three measurement directions, Bob can estimate the angles $\theta_{x}$, $\theta_{y}, \theta_{z}$ between his three axes $x, y, z$ and Alice's $z$ axis [see Fig.1(a)]. Then Bob obtains the direction of Alice's $z$ axis with a certain accuracy. To detect eavesdropping, Bob checks whether the sum of the three angles' squared cosines differs from 1 by more than an allowable error.

Indeed, if Eve stays in the quantum channel and performs blindly some measurements on the transmitted particles to extract information of Alice's $z$ axis, her eavesdropping would result in a depolarization of the spins and it follows that she would be detected by Bob (the three angles recovered by Bob would be inconsis-

*Email: gaofei_bupt@hotmail.com or hzpe@sohu.com

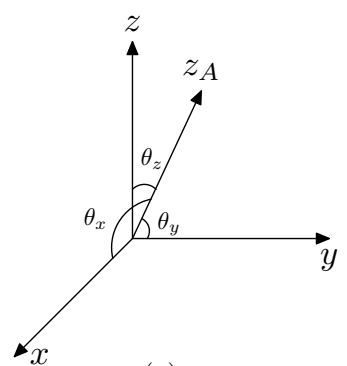

(a)

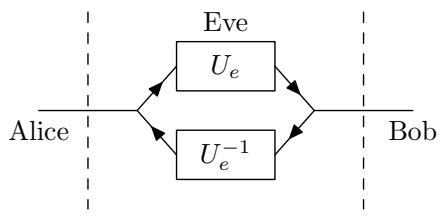

(b)
FIG. 1: (a) The way to estimate the direction of Alice's $z$ axis $z_{A}$. The three angles satisfy $\cos ^{2} \theta_{x}+\cos ^{2} \theta_{y}+\cos ^{2} \theta_{z}=1$. (b) Eve's additional strategy to avoid a general prepare-andmeasure detection.

tent). However, Eve can take a special strategy to attack. That is, she performs a unitary operation $U_{e}$ on each transmitted particle to rotate the spins by a certain angle. Eve does not know the value of this angle because she does not know what on earth the operator $U_{e}$ is in Alice's representation (therefore the choice of $U_{e}$ are at random). But she need not know it. Here the purpose of Eve is to destroy the communication instead of to extract information of the transmitted direction (In fact, without the key bits shared between Alice and Bob, Eve can never elicit any information about the transmitted direction by measurements on these qubits, which is very similar to that in one-time pad). Since the role of this attack is just rotate the spins by a same angle, Bob will obtain a changed direction of Alice's $z$ axis and, more seriously, he cannot detect the presence of Eve (obviously for any direction, the three angles $\eta_{x}, \eta_{y}, \eta_{z}$ between it and the three axes $x, y, z$ satisfy $\left.\cos ^{2} \eta_{x}+\cos ^{2} \eta_{y}+\cos ^{2} \eta_{z}=1\right)$.

Similar risk exists in other three protocols in Ref. [3], where Alice would transmit a Cartesian frame to Bob secretly. Consider any state $|\psi\rangle$ Alice will send to Bob. Since $|\psi\rangle$ is generated by Alice according to her Cartesian frame, from Bob's point of view all spins are rotated by a certain rotation $g \in S U(2)$ which connects his axes with that of Alice. Therefore, Bob's aim is to infer $g$ by measurements on all the states Alice sent to him. This 
is the main idea of the three protocols. Here Eve can also perform a unitary operation $U_{e}$ on each particle (or a collective rotation $U_{e}^{\otimes N}$ ). Again, Eve just chooses a unitary operation at random and does not know the value of $e$ referring to Alice's Cartesian frame. Thus, after some measurements, Bob's inferred parameter, which connects Alice and Bob's axes, would be eg but not $g$. Apparently, it looks like that the parameter Alice sent is indeed eg instead of $g$, which is the only difference when this attack happens. So, everything goes naturally. As a result, Bob obtains a Cartesian frame different from Alice's and Eve would not be detected.

It can be seen that this special attack causes a severe effect, that is, the RF cannot be successfully shared between Alice and Bob as they intended. More seriously, in the end of the protocol, when Alice and Bob are congratulated for the successful sharing of the Cartesian frame, they even do not know they have been cheated by Eve. When Alice and Bob utilize the different RFs to distribute secret key bits [4], remarkable errors would appear. But at that time they still do not know whether the RFs are inconsistent or an eavesdropper exists in the process of key distribution, which is a really intractable business. Consequently, this problem must be overcome in a real implementation. In fact, the above attack is a special kind of denial-of-service attack, and it is also forbidden in a quantum secure direct communication (QSDC) protocol [5, 6].

To communicate something secretly, as generally required in quantum cryptography, we have to take all thinkable attack strategies into consideration. Otherwise the intended communications may be attacked successfully (for instances, see Refs. [6, 7], 8, 9, 10, 11, 12]). In Ref. [3] the main attention is paid to forbid Eve to extract information of the transmitted RF, while the consistency of the two RFs is overlooked.

Now consider how to detect this special attack. Different from a general protocol of quantum cryptography, Alice and Bob have not a shared RF before the communication. Therefore, the ability of their possible ways to check eavesdropping is limited. For example, as described in Ref. [3], they can only check the uniqueness of the transmitted direction or frame, or employ the rotationally invariant subsystems of the test qubits to detect. But both of them are useless for the special attack. Another immediate manner to detect is to compare the consistency of Alice and Bob's RFs at the end of the communication. In a QKD protocol Alice and Bob can sample some of the key bits to check if the two keys are identical publicly. However, this strategy cannot be used here because the transmitted object is unspeakable information which cannot be discussed directly in a classical channel.

An alternative way to detect the special attack is using the general prepare-and-measure model. In particular, Alice (Bob) prepares a certain state and sends it to Bob (Alice). After the measurement of Bob (Alice), they can judge the consistency of their RFs by comparing the ini- tial state and the measurement result in public. However, this strategy would not work if Eve does an additional trick. That is, Eve performs the unitary operation $U_{e}$ on all the qubits from Alice to Bob and another one $U_{e}^{-1}$ on all the qubits from Bob to Alice [see Fig.1(b)]. As discussed in above paragraphs, the difference of Alice and Bob's RFs is just $U_{e}$ (under the above attack). As a result, under Eve's operations $U_{e}$ and $U_{e}^{-1}$, the prepared state by Alice (Bob) and the measured state by Bob (Alice) would exhibit the same physical features referring to their respective RFs. Thus, no errors would appear when Alice and Bob do their comparison.

In view of the above analysis, to expose the special attack, the check qubits should not be transmitted in the communication channel. A possible way is to use some states which are previously shared between Alice and Bob. For example, Alice and Bob share some entangled pairs in the spin singlet state $\left|\Psi^{-}\right\rangle=(|01\rangle-|10\rangle) / \sqrt{2}$ before the communication of RF (that is, for each pair, Alice holds one photon and Bob controls the other). When a certain RF has been transmitted Alice and Bob measure the spins of each shared singlet state referring to their respective RFs (the RF Alice sent and the one Bob received). Afterwards they compare the measurement results publicly. If two RFs are identical the results should be determinately anti-correlated. By this way, Alice and Bob can assure the consistency of the two RFs (with certain accuracy) when the error rate of this comparison is low enough. However, there is a problem in this detection. That is, the requirement of shared singlet pairs is too strong, with which Alice and Bob can even achieve the whole transmission of a private RF [13]. Obviously, if Alice measures the spins of her photons in the shared singlet pairs referring to her RF and announces the results, Bob can obtain the RF by the technique similar to that in the first protocol in Ref. [3].

One may argue that Eve can do the same sort of attack in many other situations (for example, one-time pad, QKD, and QSDC) and consequently it is not so meaningful to discuss this problem further here. However, it is not the fact for the case of RF sharing. As far as this attack is concerned, the transmission of an RF and that of a classical or quantum message are quite different. More specifically, when a message is transmitted, this kind of attack can be easily avoided by the manners of message authentication, error correction code, or directly sampling some random transmitted bits/qubits to check eavesdropping [6]. But all these manners are useless for the transmission of an RF. From the above analysis we can see that this problem is intractable and far from being totally resolved. It is urgent to find an effective way to detect this attack (or check the consistency of the shared RFs) in the transmission of an RF. As a result, though this problem can be accepted as inevitable in previous cryptographic models such as one-time pad, QKD, and QSDC, it should be paid more attention in the case of RF sharing.

In conclusion, we present a special attack to the proto- 
cols of secret communication of RF, by which an eavesdropper can change the transmitted RF without being detected. It means that the obtained RF should be reexamined in such protocols. Furthermore, the way to check the consistency of the two RFs is discussed though it needs more further study. Note that the communication of $\mathrm{RF}$ is a new topic and it may interest many scholars. We hope that the special attack is noticed and taken into account in the following research.

We are grateful to the anonymous reviewer for helpful comments. This work is supported by the National
High Technology Research and Development Program of China, Grant No. 2006AA01Z419; the National Natural Science Foundation of China, Grant Nos. 90604023, 60373059; the National Research Foundation for the Doctoral Program of Higher Education of China, Grant No. 20040013007; the National Laboratory for Modern Communications Science Foundation of China, Grant No. 9140C1101010601; the Natural Science Foundation of Beijing, Grant No. 4072020; and the ISN Open Foundation.
[1] S. D. Bartlett, T. Rudolph, and R. W. Spekkens, Rev. Mod. Phys. 79, 555 (2007).

[2] N. Gisin, G. Ribordy, W. Tittel, et al., Rev. Mod. Phys. 74, 145 (2002).

[3] G. Chiribella, L. Maccone, and P. Perinotti, Phys. Rev. Lett. 98, 120501 (2007).

[4] S. D. Bartlett, T. Rudolph, and R. W. Spekkens, Phys. Rev. A 70, 032307 (2004).

[5] K. Boström, and T. Felbinger, Phys. Rev. Lett. 89, 187902 (2002).

[6] Q. Y. Cai, Phys. Rev. Lett. 91, 109801 (2003).

[7] Y. S. Zhang, C. F. Li, and G. C. Guo, Phys. Rev. A 63, 036301 (2001).

[8] A. Wójcik, Phys. Rev. A 71, 016301 (2005).
[9] F. Gao, F. Z. Guo, Q. Y. Wen, et al., Phys. Rev. A 72, $036302(2005)$.

[10] F. Gao, F. Z. Guo, Q. Y. Wen, et al., Phys. Rev. A 72, 066301 (2005).

[11] F. G. Deng, X. H. Li, H. Y. Zhou, et al., Phys. Rev. A 72, 044302 (2005).

[12] F. Gao, Q. Y. Wen, and F. C. Zhu, Phys. Lett. A 360, 748 (2007).

[13] In the discussion, L. Maccone pointed out that "checking the consistency of the RFs to a certain degree of accuracy consumes exactly the same number of entangled pairs as exchanging an RF to the same degree of accuracy", and the details would appear on the quant-ph archive. 